\title{
Caracterização físico-quimica de farinhas oriundas de variedades de mandioca utilizadas no vale do Juruá, Acre
}

\author{
Joana Maria Leite de SOUZA¹, Virgínia de Souza ÁLVARES ${ }^{1}$, Felícia Maria Nogueira LEITE², Fabiana Silva \\ REIS², Francisco Álvaro Viana FELISBERTO³.
}

\begin{abstract}
RESUMO
Algumas características de farinha de mandioca dependem da variedade da raiz utilizada para o processamento. Objetivouse avaliar as características físico-químicas da farinha de mandioca oriundas de variedades utilizadas no estado do Acre. Os tratamentos foram compostos pelas variedades: $\mathrm{T} 1=$ Paxiubão, $\mathrm{T} 2=\operatorname{Im} 221, \mathrm{~T} 3=$ Caboquinha, $\mathrm{T} 4$ = Araçá, $\mathrm{T} 5=$ Colonial, T6 = Branquinha, T7 = Panati e T8 = Mansa e Brava. Foram coletadas amostras das oito variedades de mandioca em casas-defarinha no município de Cruzeiro do Sul, Acre. As raízes foram transportadas via aérea para o Laboratório de Tecnologia de Alimentos da Embrapa-AC, na cidade de Rio Branco, Acre. Foram avaliados: teor de umidade, cinzas, lipídios, proteína, fibra bruta, carboidratos, acidez, $\mathrm{pH}$ e atividade de água. Todas as amostras se apresentaram de acordo com os padróes estabelecidos pela Legislação Brasileira para farinha de mandioca quanto ao teor de umidade, cinzas e carboidratos. As farinhas analisadas apresentam teores baixos de fibras e baixa acidez. A atividade de água das farinhas analisadas esteve abaixo do limite mínimo capaz de permitir o desenvolvimento de microrganismos. As variedades Araçá, Colonial e Branquinha se mostraram adequadas para a fabricação de farinha de mandioca devido, principalmente, ao elevado teor de proteína e carboidratos presente nas farinhas produzidas.
\end{abstract}

PALAVRAS-CHAVE: farinha de mandioca, qualidade, variedades.

\section{Physicochemical characteristics of flours deriving of cassava varieties used in the Vale do Juruá, Acre}

\begin{abstract}
Some characteristics of the cassava depend on the variety used for the processing. The objectified of this study was to evaluate physicochemical characteristics of the cassava flour from varieties used in Acre. The treatments were composed by the varieties: T1 = Paxiubão, T2 = Im221, T3 = Caboquinha, T4 = Araçá, T5 = Colonial, T6 = Branquinha, T7 = Panati and T8 = Mansa e Brava. Samples of flour of eight cassava varieties were collect in cassava mils in Cruzeiro do Sul. The samples were sent by airmail to Rio Branco, Acre, to be analyzed in the Food and Technology Laboratory at Embrapa-AC. The evaluated parameters were: water percentage, ashes, lipids, protein, fiber, carbohydrates, acidity, $\mathrm{pH}$ and water activity. All samples were taken according to the established patterns of the Brazilian Legislation for cassava flour, considering water percentage, ashes and carbohydrates. The analyzed flours presented low fibers levels and low acidity. The water activity of the analyzed flours was below the minimum limit necessary to allow microorganisms development. The varieties Araçá, Colonial and Branquinha were adequate to cassava flour manufacturing, mainly because of the high protein and carbohydrates levels present in the produced flour.
\end{abstract}

KEYWORDS: cassava flour, quality, variety.

\footnotetext{
${ }_{1}^{1}$ Embrapa Acre - CPAF-AC. BR 364, Km 14, 69908-970. Rio Branco - Acre, Brasil, joana@cpafac.embrapa.br, virginia@cpafac.embrapa.com.br.

2 Universidade Federal do Acre - UFAC. Rio Branco - Acre, Brasil, felicialeite@hotmail.com , fabianasilvareis@hotmail.com

${ }^{3}$ Embrapa Acre - CPAF-AC. BR 364, Km 14, 69908-970. Rio Branco - Acre, Brasil. alvaro@cpafac.embrapa.br
} 


\section{INTRODUÇÃO}

A mandioca (Manihot esculenta, Crantz) desempenha importante papel econômico e social para o Estado do Acre, pois se constitui num dos produtos básicos da dieta alimentar da população (Moura e Cunha, 1998).

Devido ao elevado teor de umidade das raízes de mandioca recém-colhidas, em torno de $60 \%$, este produto é classificado como perecível ficando sua conservação restrita há algumas horas após a colheita (Ferreira Neto et al., 2003). Desta forma a utilização por períodos de tempo mais longos se dá através de produtos desidratados, reduzindo-se o teor de umidade para níveis que impeçam o desenvolvimento microbiano com consequiente deterioração do produto. Estes produtos desidratados são, principalmente, os diversos tipos de farinhas de mandioca largamente empregadas na alimentação humana (Vilela, 1987).

Entretanto, algumas características físico-químicas da farinha de mandioca dependem da variedade utilizada para o processamento. Chisté et al. (2006) citam que características como cinzas, proteínas e lipídeos podem variar entre as amostras de farinha devido às características intrínsecas das raízes da mandioca. Aryee et al. (2006) relacionam o teor elevado de amido encontrado na farinha de mandioca ao uso de variedades com esse teor também elevado. Além disso, é interessante citar que, também na fabricação da farinha, devem ser utilizadas variedades resistentes a doenças e altamente produtivas.

As cultivares de mandioca são classificadas em dois grupos segundo a quantidade de glicosídeos cianogênicos (açúcares) geradores de HCN (ácido cianídrico) quando em presença de determinadas enzimas do estômago: mandiocas mansas ou macaxeira, com pouco $\mathrm{HCN}$ e mandiocas bravas ou mandioca, com maiores conteúdos de HCN (Schwengber et al., 2005). As raízes dos genótipos de mandioca com elevados teores de HCN são destinadas à fabricação de farinha, enquanto as com baixos conteúdos deste ácido (abaixo de $50 \mathrm{mg} / \mathrm{kg}$ de polpa de raízes frescas) são consumidas cozidas, fritas, na forma de bolos e outras modalidades (Mendonça et al., 2003). As mandiocas mansas não são utilizadas na fabricação de farinhas, pois, segundo Carvalho et al. (1995), originam um produto com sabor adocicado, de pouca aceitação no mercado. Entretanto, como a secagem (pelo calor do sol ou em dessecadores) elimina o ácido por volatilização (Schwengber, 2005), o processamento pode ser utilizado tanto com variedades mansas como bravas (Mattos e Cardoso, 2003).

Para a produção de farinha e amido, na maioria das regiōes do Brasil é importante que as cultivares apresentem raízes com polpa, córtex e película de coloração branca, ausência de cintas nas raízes, película fina e raízes grossas e bem conformadas, o que facilita o descascamento e garante a qualidade do produto final. Apesar de se adaptar aos mais diferentes ecossistemas, a mandioca apresenta uma alta interação do genótipo com o ambiente, ou seja, os cultivares apresentam adaptação específica a determinadas regiôes e dificilmente uma mesma cultivar se comporta de forma semelhante em todos os ecossistemas (Mattos e Cardoso, 2003). O ideal é que a cultivar de mandioca apresente pelo menos $30 \%$ de amido para ser industrializada (Conceição, 1979).

No Estado do Acre, os cultivares Araçá e Panati foram lançadas pela Embrapa Acre (CPAF-AC) por apresentarem uma série de características agronômicas superiores a outras analisadas, como elevada capacidade produtiva, resistência a podridão radicular e elevado potencial para o mercado. A cultivar Panati apresenta elevado teor de amido e teor médio de ácido cianídrico nas raízes, sendo recomendada para a indústria. A cultivar Araçá possui elevado teor de amido e baixo teor de ácido cianídrico nas raízes, o que permite a sua utilização tanto na alimentação humana como na fabricação de farinha (Moura e Cunha, 1998). Outras cultivares muito utilizadas na região são também adequadas por serem consideradas resistentes (Branquinha, IM 221 e Paxiubão) ou moderadamente resistentes (Caboquinha) à podridão radicular (Moura e Silva, 1997). As variedades Branquinha, Colonial e Paxiubão apresentam teores elevados de amido, sendo particularmente importantes na industrialização (Mendonça et al., 2003).

Com base nestas observaçōes, o objetivo deste trabalho foi avaliar as características físico-químicas das farinhas oriundas de variedades de mandioca utilizadas no estado do Acre.

\section{MATERIAL E MÉTODOS}

As amostras de farinha, de oito variedades de mandioca, coletadas, em maio de 2007, em casas-de-farinha no vale do Juruá, Acre, foram produzidas de forma artesanal em forno a lenha, com chapa de ferro, sem sistema de controle de temperatura, com revolvimento manual da massa com auxílio de rodos. Os tratamentos foram compostos pelas variedades: T1 $=$ Paxiubão, T2= Im221, T3 = Caboquinha, T4 = Araçá, T5 = Colonial (MX26), T6 = Branquinha, T7 = Panati e T8 = Mansa e Brava. As amostras foram acondicionadas em sacos plásticos de $1 \mathrm{~kg}$ de capacidade e transportadas via aérea para a cidade de Rio Branco, Acre, onde foram encaminhadas para o Laboratório de Tecnologia de Alimentos da Empresa Brasileira de Pesquisa Agropecuária (Embrapa-Acre).

No laboratório as amostras foram encaminhadas para as análises bromatológicas realizadas em triplicata. $\mathrm{O}$ teor de umidade foi determinado de acordo com o método 31.1.02 da AOAC (1995), usando-se estufa a $105^{\circ} \mathrm{C}$ por 8 horas. Para o teor de cinzas, as amostras foram carbonizadas até cessar a liberação de fumaça e, posteriormente, calcinadas em mufla a $540{ }^{\circ} \mathrm{C}$ até peso constante, segundo o método 31.1.04 da AOAC (1995). O teor de lipídeo foi obtido por extração em 
Soxhlet durante $10 \mathrm{~h}$ e posterior evaporação do éter dietílico anidro, de acordo com o método 31.4.02 da AOAC (1995). Para a determinação do teor de proteínas, foi utilizada a técnica do destilador micro-Kjeldahl e bloco digestor, baseado em hidrólise e posterior destilação da amostra, utilizando o fator $6,25 \mathrm{x} \% \mathrm{~N}$, de acordo com o método 31.1.08 da AOAC (1995). Já o teor de fibra bruta foi determinado por meio de digestão do material em solução de $\mathrm{H}_{2} \mathrm{SO}_{4}$ a $1,25 \% \mathrm{p} / \mathrm{v}$ por 30 minutos, seguida de $\mathrm{NaOH} 1,25 \% \mathrm{p} / \mathrm{v}$ por mais 30 minutos, de acordo com AOAC (1995). O teor de carboidratos foi estimado por diferença, subtraindo de 100 o somatório de proteínas, lipídios, cinzas, umidade e fibra alimentar total e os resultados expressos em percentual, segundo Normas Analíticas do Instituto Adolfo Lutz (1976). A acidez total titulável foi determinada de acordo com o método 942.15 da AOAC (1995). Para o $\mathrm{pH}, 9 \mathrm{~g}$ da amostra foram misturadas em $60 \mathrm{~mL}$ de água destilada, homogeneizados e deixados em repouso por 30 minutos. A leitura foi feita em medidor de $\mathrm{pH}$ digital. E por fim, foi determinada a atividade de água em medidor de atividade de água portátil, modelo Pawkit, marca Decagon.

$\mathrm{O}$ experimento foi analisado segundo um delineamento inteiramente casualizado, com 8 tratamentos (variedades), 3 repetiçōes, sendo um saco de $1 \mathrm{~kg}$ a unidade experimental. Para cada repetição foram retiradas as médias das três replicatas antes das análises. Os dados foram submetidos à análise de variância e as médias do fator quantitativo foram comparadas pelo teste de Scott Knott a 5\% de probabilidade, através do programa computacional SAEG (Euclydes, 1993), versão 5.0.

\section{RESULTADOS E DISCUSSÃO}

De acordo com a Portaria $n^{\circ} 554$ de 30.08.1995 da Secretaria da Agricultura, do Abastecimento e Reforma Agrária (Brasil, 1995), a análise do teor de umidade, cinzas e carboidratos mostrou que todas as amostras estão de acordo com os padrōes estabelecidos pela Legislação Brasileira para farinhas de mandioca, que estabelece índice máximo de 13\% para umidade, $1,5 \%$ de cinzas e índice mínimo de $70-75 \%$ para carboidratos (Tabela 1).

Entre as farinhas analisadas, verificou-se que T7 (variedade Panati) apresentou o maior teor de umidade e os tratamentos T5 e T6, variedades Colonial e Branquinha, respectivamente, apresentaram os menores valores médios quanto a esta característica. Os valores de umidade das amostras estão abaixo dos encontrados por Ferreira Neto et al. (2003) e Aryee et al. (2006), que encontraram um teor médio de umidade de 8,00 $\pm 0,33 \%$ e $8,19 \pm 0,93 \%$, respectivamente, ao estudarem as características físico-químicas de farinhas produzidas no sul do estado de Santa Catarina e em Gana.
Tabela 1 - Valores médios (b.s.), em percentagem, do teor de umidade (U), cinzas (CZ), proteína bruta (PB), lipídeos (LI), fibra bruta (FB) e carboidratos totais (CB) na farinha de mandioca de variedades utilizadas no Acre

\begin{tabular}{lllllll}
\hline Tratamento $^{1}$ & $\mathrm{U}$ & $\mathrm{CZ}$ & $\mathrm{LI}$ & $\mathrm{PB}$ & $\mathrm{FB}$ & $\mathrm{CB}$ \\
\hline 1 & $3,73 \mathrm{c}$ & $0,33 \mathrm{~b}$ & $1,06 \mathrm{~d}$ & $0,54 \mathrm{c}$ & $1,93 \mathrm{a}$ & $92,47 \mathrm{~b}$ \\
\hline 2 & $4,76 \mathrm{~b}$ & $0,65 \mathrm{a}$ & $1,24 \mathrm{c}$ & $0,64 \mathrm{~b}$ & $2,01 \mathrm{a}$ & $90,82 \mathrm{~d}$ \\
\hline 3 & $4,45 \mathrm{~b}$ & $0,80 \mathrm{a}$ & $1,17 \mathrm{c}$ & $0,86 \mathrm{a}$ & $1,89 \mathrm{a}$ & $90,92 \mathrm{~d}$ \\
\hline 4 & $4,13 \mathrm{~b}$ & $0,76 \mathrm{a}$ & $1,26 \mathrm{c}$ & $0,88 \mathrm{a}$ & $1,68 \mathrm{~b}$ & $90,88 \mathrm{~d}$ \\
\hline 5 & $3,13 \mathrm{~d}$ & $0,66 \mathrm{a}$ & $1,57 \mathrm{~b}$ & $0,80 \mathrm{a}$ & $1,74 \mathrm{~b}$ & $92,24 \mathrm{~b}$ \\
\hline 6 & $2,70 \mathrm{~d}$ & $0,81 \mathrm{a}$ & $1,18 \mathrm{c}$ & $0,49 \mathrm{c}$ & $2,05 \mathrm{a}$ & $92,72 \mathrm{a}$ \\
\hline 7 & $5,89 \mathrm{a}$ & $0,51 \mathrm{~b}$ & $1,61 \mathrm{~b}$ & $0,31 \mathrm{~d}$ & $1,69 \mathrm{~b}$ & $89,83 \mathrm{e}$ \\
\hline 8 & $4,27 \mathrm{~b}$ & $0,55 \mathrm{~b}$ & $1,86 \mathrm{a}$ & $0,30 \mathrm{~d}$ & $1,66 \mathrm{~b}$ & $91,39 \mathrm{c}$ \\
\hline
\end{tabular}

${ }^{\top} \mathrm{T} 1$ = Paxiubão; $\mathrm{T} 2=\operatorname{Im} 221 ; \mathrm{T} 3$ = Caboquinha; $\mathrm{T} 4=$ Araçá; $\mathrm{T} 5=$ Colonial; $\mathrm{T} 6=$ Branquinha; $\mathrm{T7}=$ Panati e T8 = Mansa e Brava

As médias seguidas por uma mesma letra na coluna não diferem estatisticamente entre si pelo teste de Scott Knott a $5 \%$ de probabilidade.

A umidade é um importante parâmetro no armazenamento da farinha de mandioca, sendo que níveis maiores que $12 \%$ podem proporcionar o crescimento de microrganismos. Dessa forma, baixos níveis são favoráveis para oferecer condições para uma maior vida-de-prateleira do produto. $\mathrm{O}$ teor de umidade da farinha de mandioca está relacionado com o seu processo de fabricação (Chisté et al., 2006), podendo variar, principalmente com o tempo e temperatura de tostagem, o que não foi mensurado por fazer parte de um processo de fabricação artesanal.

Na legislação brasileira (Brasil, 1995) conceitua-se as cinzas como o resíduo mineral fixo resultante da incineração da amostra do produto. Desta forma, o teor de cinzas da farinha de mandioca pode estar relacionado tanto com as características intrínsecas das raízes (Chisté et al., 2006), quanto com o processo de fabricação, como, por exemplo, o descascamento. De acordo com Paiva (1991), valores maiores que a tolerância máxima permitida pode ser também um indicativo de teores significativos de $\mathrm{Ca}, \mathrm{P}, \mathrm{Fe}$ e $\mathrm{Mg}$, como também, mais provavelmente, indicam contaminação por material estranho ao produto ocasionado por falhas em algumas etapas do processamento.

Nas amostras analisadas, os menores teores de cinzas foram obtidos nos tratamentos T1 (Paxiubão), T7 (Panati) e T8 (Mansa e Brava), não diferindo estatisticamente entre si (Tabela 1). Os demais tratamentos tiveram valores mais elevados para esta característica. Todas as amostras avaliadas foram inferiores aos valores médios de minerais encontrados por Ferreira Neto et al. (2003) de 1,70\%, não sendo citado pelos autores se foram utilizados descascadores industriais e se houve remoção do feixe lenhoso ou cepa da raiz. No caso da farinha oriunda do Acre, um dos fatores que pode ter interferido nesse menor valor é a produção artesanal, onde as mandiocas são descascadas manualmente, sendo possível 
remover película, feloderma e fibra central, além do amido não ser retirado para a fabricação de gomas.

Não há referências com relação aos teores de lipídeos na farinha de mandioca na legislação brasileira (Brasil, 1995). Contudo, sabe-se que tal característica pode variar devido às características intrínsecas das raízes da mandioca (Chisté et al., 2006).

O tratamento 8 (Mansa e Brava) se sobressaiu dentre os demais pelo maior teor de lipídeos encontrado na farinha de mandioca oriundo desta variedade (Tabela 1), enquanto que no tratamento 1 (variedade Paxiubão) foi obtido o menor teor de lipídeos dentre todos os tratamentos analisados.

Apenas no tratamento constituído pela variedade Mansa e Brava (T8) o valor de lipídeos foi superior ao relatado por Ferreira Neto et al. (2003) de 1,71\%.

As farinhas apresentam um teor de proteínas bastante baixo, o que já é esperado frente à composição da raiz da mandioca (Albuquerque et al., 1993). Visto isto, este produto é considerado um alimento essencialmente energético em função do baixo teor de proteína e elevado teor de carboidratos.

Os valores de proteína obtidos nas amostras de farinha variaram de $0,30 \%$ a $0,88 \%$, com as maiores médias para os tratamentos 3 (Caboquinha), 4 (Araçá) e 5 (Colonial) (Tabela 1). A proteína encontrada na farinha de mandioca está diretamente relacionada à variedade da raiz a que lhe deu origem (Chisté et al., 2006). Desta forma, uma variedade de mandioca com maior teor de proteínas é indicada para a fabricação da farinha. Não obstante, foram obtidos para as variedades Panati (T7) e Mansa e Brava (T8) os menos teores de proteína dentre as amostras analisadas.

Os resultados obtidos foram inferiores aos obtidos por Ferreira Neto et al. (2003) de 1,70\%. Dias e Leonel (2006) encontraram valores de $0,57 \%$ a $1,08 \%$ de proteína na farinha de mandioca de diferentes localidades do Brasil, e Chisté et al. (2006) valores de $0,53 \%$ a $0,93 \%$ na farinha de mandioca produzida no Pará.

Quanto ao teor de fibras, a Legislação Brasileira não estipula valores, contudo, foi possível verificar diferenças significativas deste componente entre as farinhas analisadas. A farinha dos tratamentos T1 (Paxiubão), T2 (Im221), T3 (Caboquinha) e T6 (Branquinha) foram as que apresentaram os maiores teores de fibras, sendo que os demais tratamentos tiveram as menores médias (Tabela 1).

Mattos e Martins (2000) classificaram os alimentos de acordo com o seu teor de fibras: baixo (inferior a 2,4 $\mathrm{g}$ fibras $/ 100 \mathrm{~g}$ ), moderado $(2,4 \mathrm{a} 4,4 \mathrm{~g}$ fibras $/ 100 \mathrm{~g}$ ) ou alto (superior a 4,4 g fibras/ $100 \mathrm{~g}$ ). De acordo com essa classificação, todas as farinhas analisadas apresentaram teores baixos de fibras (Tabela 1).
Acredita-se que as fibras exercem suas funções gastrointestinais através de sua ação física, capacidade de hidratação e de aumentar o volume e a velocidade de trânsito do bolo alimentar e fecal. As fibras possuem também capacidade de complexar-se com outros constituintes da dieta por meio de vários mecanismos, podendo arrastá-los em maior quantidade na excreção fecal. Dessa forma, tanto nutrientes essenciais, proteínas, minerais e vitaminas, como substâncias tóxicas, poderão ser excretadas em maior ou menor quantidade, dependendo da qualidade e da quantidade da fibra presente na dieta (Raupp et al., 1999).

Os valores de carboidratos (Tabela 1) foram superiores aos valores médios encontrados por Ferreira Neto et al. (2003) de $89 \%$ e dos teores mínimos exigidos por Brasil (1995) de 70-75\%. Dias e Leonel (2006) encontraram valores de $81,02 \%$ a $91,56 \%$ de amido na farinha de mandioca. Os valores encontrados, como sugerido por Aryee et al. (2006), indicam que foram utilizadas variedades com elevado teor de amido e poderiam ser usadas para diversos produtos comerciais como derivados de amido, álcool e glicose. Entre as amostras analisadas, a farinha oriunda da variedade Branquinha (T6) apresentou o maior teor (92,72\%), diferindo significativamente das demais, e as farinhas provenientes das variedades Im221 (T2), Caboquinha (T3) e Araçá (T4) tiveram as menores médias.

Dentre as características analisadas, a acidez se destaca como uma das mais importantes, estando relacionada com o processo de fabricação da farinha, como por exemplo, o tempo de fermentação ou tempo de prensagem da massa de mandioca triturada.

A amostra de farinha do tratamento $\mathrm{T} 5$ teve a maior acidez dentre as demais, ultrapassando inclusive o limite máximo permitido pela legislação brasileira de $3 \%$ para farinha de mandioca (Brasil, 1995), e os tratamentos 2 e 7 tiveram as menores médias (Tabela 2).

Tabela 2 - Valores médios de acidez (meq $\mathrm{NaOH} / 100 \mathrm{~g}$ ), pH e atividade de água da farinha de mandioca de variedades do Acre

\begin{tabular}{llll}
\hline Tratamento & Acidez & $\mathrm{pH}$ & Atividade de água \\
\hline T1 & $2,82 \mathrm{~b}$ & $5,47 \mathrm{~b}$ & $0,09 \mathrm{~g}$ \\
T2 & $1,28 \mathrm{~d}$ & $5,02 \mathrm{c}$ & $0,19 \mathrm{~b}$ \\
T3 & $2,19 \mathrm{c}$ & $4,73 \mathrm{~d}$ & $0,16 \mathrm{~d}$ \\
T4 & $1,84 \mathrm{c}$ & $5,09 \mathrm{c}$ & $0,15 \mathrm{e}$ \\
T5 & $3,72 \mathrm{a}$ & $4,54 \mathrm{e}$ & $0,11 \mathrm{f}$ \\
T6 & $2,69 \mathrm{~b}$ & $5,05 \mathrm{c}$ & $0,09 \mathrm{~g}$ \\
T7 & $1,20 \mathrm{~d}$ & $6,15 \mathrm{a}$ & $0,29 \mathrm{a}$ \\
T8 & $2,08 \mathrm{c}$ & $5,41 \mathrm{~b}$ & $0,17 \mathrm{c}$ \\
\hline
\end{tabular}

${ }^{\top} \mathrm{T} 1=$ Paxiubão; $\mathrm{T} 2=\operatorname{Im} 221 ; \mathrm{T} 3=$ Caboquinha; $\mathrm{T} 4=$ Araçá; $\mathrm{T} 5=$ Colonial; $\mathrm{T} 6=$ Branquinha; 77 = Panati e T8 = Mansa e Brava. 
As médias seguidas por uma mesma letra na coluna não diferem estatisticamente entre si pelo teste de Scott Knott a $5 \%$ de probabilidade.

Os resultados de acidez foram inferiores aos valores encontrados por Chisté et al. (2006) com variações nas amostras de 4,11 a 7,10. A acidez elevada pode ser indicativa de falta de higiene no processo, o que é uma característica de processos artesanais (Dias e Leonel, 2006). A exposição prolongada da massa de mandioca à temperatura ambiente elevada e o aumento no tempo de fermentação favorece, consequentemente, o aumento na acidez.

$\mathrm{O}$ pH também é um fator de grande importância na limitação da capacidade de desenvolvimento de microrganismos no alimento. Em função deste parâmetro, de acordo com Soares et al. (1992), os alimentos podem ser classificados em: pouco ácidos (pH >4,5), ácidos $(4,5$ a 4,0) e muito ácidos $(<4,0)$. Diante desta classificação, as amostras de farinha analisadas foram consideradas pouco ácidas, sendo que a farinha do tratamento $\mathrm{T} 7$ teve $\mathrm{pH}$ estatisticamente maior que as demais (Tabela 2). Cuidados com a higiene durante o processo são essenciais para um produto final de qualidade, já que, de acordo com Soares et al. (1992), a maioria das bactérias, dos fungos filamentosos e das leveduras cresce em $\mathrm{pH}$ superior a 4,5.

A atividade de água tem sido considerada como uma propriedade fundamental no controle de qualidade de alimentos. De acordo com Chisté et al. (2006), considera-se a atividade de água de 0,60 como o limite mínimo capaz de permitir o desenvolvimento de microrganismo, daí o fato de os alimentos desidratados, como a farinha de mandioca, serem considerados microbiologicamente estáveis.

Neste trabalho, as amostras em estudo apresentaram atividade de água na faixa de 0,09 a 0,29 (Tabela 2). Ferreira Neto et al. (2005) encontraram valores de atividade de água de 0,38 a $0,49 \mathrm{em}$ amostras de farinha de mandioca temperadas e embaladas em sacos plásticos, durante o armazenamento. Já Chisté et al. (2006), valores de 0,31 a 0,61 em amostras de farinha de mandioca seca.

Cereda e Vilpoux (2003) citam que as únicas composições que dependem do processo e do tipo de armazenamento da farinha são a umidade e a acidez. Por outro lado, características como cinzas, lipídeos, proteínas (Chisté et al., 2006) e carboidratos (Aryee et al., 2006) podem estar relacionadas com as características intrínsecas das raízes. De acordo com estas informaçôes e com os dados obtidos, as variedades Caboquinha, Araçá e Colonial são adequadas para a fabricação de farinha de mandioca devido, principalmente, ao elevado teor de proteína, podendo auxiliar na qualidade nutricional da alimentação dos moradores da região, e a variedade Branquinha pelo alto teor de carboidratos presente na farinha produzida.

\section{CONCLUSÃO}

Com relação ao teor de umidade, cinzas e carboidratos, todas as farinhas encontram-se dentro dos limites fixados pela legislação;

As farinhas analisadas apresentam teores baixos de fibras e são pouco ácidas;

Apenas a farinha do tratamento $\mathrm{T} 5$ tem o teor de acidez acima do limite permitido pela legislação;

A atividade de água das farinhas analisadas está abaixo do limite mínimo capaz de permitir o desenvolvimento de microrganismos;

As variedades Caboquinha, Araçá e Colonial são adequadas para a fabricação de farinha de mandioca por contribuir com a melhoria do teor de proteína na alimentação dos consumidores e a variedade Branquinha pelo alto teor de carboidratos presente na farinha produzida.

\section{AGRADECIMENTOS}

Aos produtores da região de Cruzeiro do Sul - Acre, pelo fornecimento da farinha de mandioca e à FINEP pelos recursos financeiros.

\section{BIBLIOGRAFIA CITADA}

Albuquerque, T. T. O.; Miranda, L. C. G.; Salim, J.; Teles, F. F. F.; Quirino, J. G. 1993. Composição centesimal da raiz de 10 variedades de mandioca (Manihot esculenta Crantz) cultivadas em Minas Gerais. Revista Brasileira de Mandioca, 12 (1): 7-12.

AOAC 1995. Association of Official Analytical Chemists. Official methods of analysis of the AOAC International. 16. edição. Arlington.

Aryee, F. N. A.; Oduro, I.; Ellis, W. O.; Afuakwa, J. J. 2006. The physicochemical properties of flour samples from the roots of 31 varieties of cassava. Food Control, 17: 916-922.

Brasil, Portaria n. 554, de 30 de agosto de 1995. 1995. Norma de identidade, qualidade, apresentação, embalagem, armazenamento e transporte da farinha de mandioca. Diário Oficial da República Federativa do Brasil, Brasília, DF.

Carvalho, P. C. L.; Fukuda, W. M. G.; Cruz, P. J.; Costa, J. A. 1995. Avaliação agronômica e tecnológica de cultivares de mandioca para consumo 'in natura'. Revista Brasileira de Mandioca, 14 (1): 7-15.

Cereda, M. P.; Vilpoux, O. F. 2003. Farinhas e derivados. In: Cereda, M.P.; Vilpoux, O.F. Tecnologia, usos e potencialidades de tuberosas amiláceas latino americanas. V. 3. Série culturas de tuberosas amiláceas latino americanas. Fundação Cargil, Campinas, São Paulo. p. 576-620.

Chisté, R. C.; Cohen, K. O.; Mathias, E. A.; Ramos Júnior, A. G. A. 2006. Qualidade da farinha de mandioca do grupo seca. Ciência e Tecnologia de Alimentos, 26 (4): 861-864.

Conceiçāo, A. J. da. 1979. A mandioca. UFBA/Embrapa/BNB/ Brascan Nordeste, Cruz das Almas. 382 pp. 
Dias, L. T; Leonel, M. 2006. Caracterização físico-química de farinhas de mandioca de diferentes localidades do Brasil. Ciência e Agrotecnologia, 30 (4): 692-700.

Euclydes, R. F. 1993. Manual de utilização do programa SAEGsistema para análises estatísticas e genéticas. UFV/CPD, Viçosa, MG. 59 pp.

Ferreira Neto, C. J.; Figueiredo, R. M. F.; Queiroz, A. J. M. 2003. Avaliação físico-química de farinhas de mandioca durante o armazenamento. Revista Brasileira de Produtos Agroindustriais, 5 (1): 25-31.

Instituto Adolfo Lutz. 1976. Normas analiticas do Instituto Adolfo Lutz: métodos químicos e fisicos para análise de alimentos. $2^{\mathrm{a}}$ edição. São Paulo, v. 1.371 pp.

Mattos, P. L. P.; Cardoso, E. M. R. 2003. Cultivo da mandioca para o estado do Pará. Embrapa Mandioca e Fruticultura, Cruz das Almas. (Sistemas de Produção, 13). (http://sistemasdeproducao. cnptia.embrapa.br/FontesHTML/Mandioca/mandioca para/ cultivares). Acesso: 31/05/2007.

Mattos, L. L.; Martins, I. S. 2000. Consumo de fibras alimentares em população adulta. Revista de Saúde Pública, 34: 50-55.

Mendonça, H. A.; Moura, G. M.; Cunha, E. T. 2003. Avaliação de genótipos de mandioca em diferentes épocas de colheita no Estado do Acre. Pesquisa Agropecuária Brasileira, 38 (6): 761-769.

Moura, G. M.; Cunha, E. T. 1998. Panati e Araçá: novas cultivares de mandioca para o cultivo na microrregiāo do Alto Purus no Estado do Acre. Embrapa, Rio Branco, AC. 4pp. (Comunicado Técnico, 86).
Moura, G. M.; Silva, M. D. O. 1997. Avaliação de resistência de cultivares de mandioca à podridão de raizes. Embrapa, Rio Branco, AC. 4pp. (Comunicado Técnico, 76).

Paiva, F. F. A. 1991. Controle de qualidade da farinha de mandioca (Manihot esculenta Crantz) produzida na regiāo metropolitana de Fortaleza. Dissertação de Mestrado, Universidade Federal do Ceará, Fortaleza, CE. 216pp.

Raupp, D.S. et al . 1999. Composição e propriedades fisiológico - nutritivas de uma farinha rica em fibra insolúvel obtida do resíduo fibroso de fecularia de mandioca. Ciência e Tecnologia de Alimentos, Campinas, 19 (2). (http://www.scielo.br/scielo. php?script=sci_arttext $\&$ pid $=$ S0101-20611999000200009\& lng=pt\&nrm= iso). Acesso em: 30 Maio 2007.

Schwengber, D. R.; Smiderle, O. J.; Mattioni, J. A. M. 2005. Mandioca: recomendaçôes para plantio em Roraima. Embrapa Roraima, Boa Vista, Roraima. 30 pp. (Circular técnica, 5).

Soares, A. G.; Freire-Júnior, R. S. 1992. Curso de higiene e sanificação na indústria de alimentos. Rio de Janeiro, Embrapa, CTAA. 97 pp.

Vilela, E. R.; Juste Junior, E. S. G. 1987. Tecnologia da farinha de mandioca. Informe Agropecuário, 145 (13): 60-62.

Recebido em 28/11/2007

Aceito em 26/06/2008 